\title{
In Dealing with Influenza Infection in Pregnant Women
}

\author{
Myat San Yi' ${ }^{1 *}$, MiMi Khaing ${ }^{1}$ and SoeLwin ${ }^{3}$ \\ ${ }^{1}$ Medical Lecturer, Obstetrics and Gynaecology Department, University Malaysia Sarawak, Kuching, Malaysia \\ ${ }^{2}$ Associate Professor, Obstetrics and Gynaecology Department, University Malaysia Sarawak, Kuching, Malaysia
}

Received: 眥 May 28, 2018; Published: 㘹June 06, 2018

*Corresponding author: Myat San Yi, Medical Lecturer, Obstetrics and Gynaecology Department, University Malaysia Sarawak, Kuching, Malaysia

\begin{abstract}
Pregnant women are vulnerable to influenza infection. Seasonal flu infection is common and the diagnosis is mainly clinical based. If clinician is not aware of warning symptoms and signs, the seasonal flu infection can lead to serious morbidity and mortality. Therefore, clinician plays an important role to observe and monitor closely and update oneself with the progress of the disease process. Simple interventions like hand hygiene, covers coughs and sneezes with isolation will be helpful to prevent the transmission of the disease.
\end{abstract}

Keywords: Influenza infection in pregnancy

Abbreviations: HA: Hemagglutinin; NA: Neuraminidase: URIs: Upper Respiratory Tract Infections; ILI : Influenza-Like Illness; ARDS: Adult Respiratory Distress Syndrome; GISRS: Global Influenza Surveillance and Response System

\section{Introduction}

Influenza (Flu) is a very common infection in general population. It is generally mild and self- recovered infection, however, very few people know the fact that it has a sophisticated assortment in the viral structure (antigenic shift) and its course of the disease itself can result in a tremendously harmful outcome. There were so far 4 pandemic attacks worldwide. Spain in 1918 (unknown strain but suggestive of avian-like $\mathrm{H}_{1} \mathrm{~N}_{1}$ ), Asian flu in 1957 by $\mathrm{H}_{2} \mathrm{~N}_{2}$, Hong Kong in 1968 and Mexico in 2009. After all the pandemic attacks, health personnel become more aware of its lethal complications as well as its after-maths. Normally, it occurs as a seasonal flu and as an endemic entity but it gets upgraded into epidemic then pandemic. For a virus, it is a single-stranded, enveloped RNA virus from Orthomyxoviridae family. Its incubation period is $2-5$ days and the transmission is mainly air-borne i.e. droplet inhalation or direct transmission or contact through hands and fomites. There are 4 types of virus (A, B, C and D). The commonest and the most virulent pathogenic group is group $\mathrm{A}$ which has well-known subtypes like $\mathrm{H}_{1} \mathrm{~N}_{1}$ (Swine flu or 2009 Pandemic flu), $\mathrm{H}_{5} \mathrm{~N}_{1}$ (2004 Avian flu), $\mathrm{H}_{3} \mathrm{~N}_{2}$ (1968 Hong Kong flu) and others. There are no other subtypes in group B, C and D. The subtypes are classified based on antibody responses to glycol proteins such as hemagglutinin (HA) and neuraminidase (NA) on the surface of the virus [1]. The clinical presentations are generally headache, fever, dry cough, sneezing, running nose, malaise, muscle ache and pain, sore throat, but one third may be asymptomatic. The cough may persist for about two weeks after other symptoms subside. The symptoms can be overlapped with those caused by other viral upper respiratory tract infections (URIs) including human para influenza virus, adenovirus, entero virus and paramyxo viruses which will cause influenza-like illness (ILI) [2].

The diagnosis is mainly clinical in most of the seasonal cases but once the illness presents with the following severe symptoms, novel influenza strain should be suspected. The course of the novel infection will last longer than its normal course and its symptoms are refractory to normal symptomatic treatment.

I. Warning signs for the clinician.

II. Difficulty breathing or shortness of breath. 
III. Pain or pressure in the chest or abdomen.

IV. Sudden dizziness.

V. Confusion.

VI. Severe or persistent vomiting.

VII. Flu-like symptoms that improve but then return with fever and worse cough.

The harmful effect is more pronounced in pregnant women, children under 5 years, elderly people, immune-compromised patients, general debility and the patients with underlying respiratory illness especially asthma. The reason for the pregnant women being prone to severe influenza infection is due to physiological changes like increased heart rate, stroke volume and 02 consumption, decreased lung capacity and a shift away from cell- mediated immunity towards humoral immunity [3]. During the 2009 pandemic flu, 10\% of pregnant women were among those admitted to the hospital in Florida, USA [4]. Even in seasonal outbreak, pregnant women are found to be affected more than non-pregnant adult women in terms of morbidities like an increase in hospital admission, increased admission to ICU and long-term hospital stays. Diagnostic test recommended is reverse transcription polymerase chain reaction (RT-PCR) test and viral culture [5]. A rapid influenza antigen test (RIATs) is another onestop useful test in our clinical practice and its use is agreed by CDC although the sensitivity is $40-69 \%$ [6].The specimens collectable are from nasal swabs, nasopharyngeal swabs, throat swabs, endotracheal tube aspirates and broncho pulmonary secretions from lavage.

The complications are higher with advancing pregnancy even result in maternal death. The severity depends on obstetric risk factors like pre-eclampsia, multiple pregnancy.., independent risk factors like obesity, smoking and medical illnesses like asthma, autoimmune disease. While looking through in all reachable case-series and case reports, there were variable severities like pneumonia, myocarditis, encephalitis and myositis with multiorgan failure (ARDS-adult respiratory distress syndrome) from sepsis leading to mortality. Effects on the pregnancy include nonreassuring fetal testing (mostly fetal tachycardia) and febrile morbidity. There are theoretical risks of congenital anomalies and neural tube defect in early pregnancy associated with hyperthermia 5. There are evidences that pregnant women can develop miscarriage with premature rupture of membrane from CDC report 7.

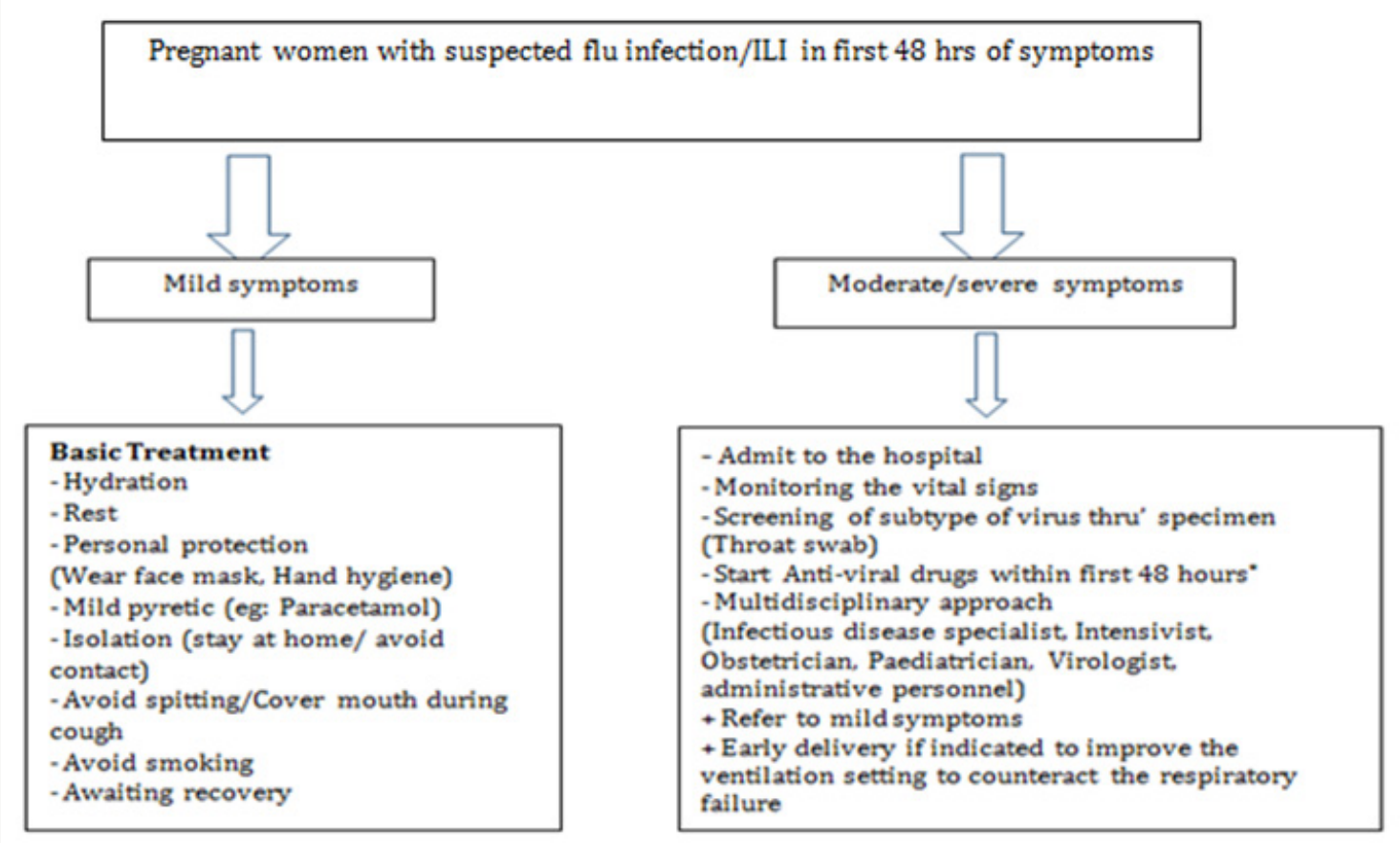

*Anti-viral drugs will help to relieve the symptoms and reduce the viral shedding, but more beneficial if starts within 12 48 hours. Neuraminidase inhibitors are used and they are as an adjunct to immunization. Oseltamivir is taken orally and zanamivir is via inhalation. Both are category $\mathrm{C}$ but Oseltamivir is preferred over zanamivir due to its well-evidenced results from clinical experience with a guaranteed systemic absorption.

\section{Figure 1.}

The treatment is mainly symptomatic but, at the same time, the woman needs to be closely monitored whether the symptoms are subsided or become more serious. Life style changes like avoid smoking with personal hygiene are considered as important measures to prevent the transmission. The women should take vivid amount of water and should be isolated from others. Surveillance on the appearance of new symptoms as well as progress of current condition is necessary during isolation period. Personal protection 
measures like avoid touching the eyes, nose or mouth; proper hand hygiene with soap and water, or with alcohol-based hand rubs, covering coughs and sneezes; avoiding close contact with sick people; and staying home during the illness will be helpful. Wearing of face mask is recommended. (Figure 1) and (Table 1) Immunization is recommended to pregnant women especially during flu season (November-March). Macdonald stated that they are low-cost interventions that have been shown to have substantial benefits for both mother and baby [8]. During 2016-17 flu seasons, an estimated $50 \%$ of pregnant women in the US were vaccinated [7]. Globally, World Health Organisation recommends the surveillance Table: 1. system through Global Influenza Surveillance and Response System (GISRS) which monitors and analyses the influenza disease progress. Center for Disease Control (CDC) [9] also observes closely the cases erupted periodically and recruits the virus particles and specimens for future use from worldwide source. In conclusion, obstetricians should be careful as their patients are vulnerable to this common infection and simple interventions like hand hygiene and personal protection will help to prevent the transmission of flu infection. All disciplines should be collaborated in the treatment of severe cases. By keeping this in mind, clinicians should respond all the calls appropriately as it is better to be safe than being sorry.

\begin{tabular}{|c|c|c|}
\hline Drug & Treatment & Chemoprophylaxis \\
\hline Oseltamivir & $75 \mathrm{mg}$ twice a day for 5 days & $75 \mathrm{mg}$ once a day for 10 days \\
\hline Zanamivir & Two $5 \mathrm{mg}$ inhalations twice per day for 5 days & Two $5 \mathrm{mg}$ inhalations daily for 10 days \\
\hline Peramivir & One time intravenously over $15-30 \mathrm{mins}$ & \\
\hline
\end{tabular}

\section{References}

1. https://en.wikipedia.org/wiki/Influenza

2. https://emedicine.medscape.com/article/

3. Katie QC, Elizabeth HH, John S (2016) Flu-like symptoms in pregnancy. Australian Family Physician 45(5): 307-308.

4. Louie JK AM, Winter K, Jean C, Gavali S, Schechter R, et al. (2009) Factors associated with death or hospitalization due to Pandemic 2009 influenza (H1N1) infection in California. JAMA 302(17): 1896-1902.

5. Amanda C, Stephen FT, Errol RN (2009) H1N1 influenza in pregnancy: What all obstetric health providers ought to know. Reviews in Obstetrics and Gynaecology 2(3): 139-145.
6. Brad JH, James HD, Carmen LL, Alan DK (2009) Novel influenza A (H1N1) viral infection in late pregnancy: Report of a case. The Ochsner Journal 10(1): 32-37.

7. https://www.cdc.gov/flu/guidelines

8. Macdonald NE, Riley LE, Steinhoff MC (2009) Influenza immunization in pregnancy. Obstet Gynaecol 114: 365-368.

9. (2009) Pregnant women and novel influenza A (H1N1) virus: Considerations for clinicians. Centers for Disease Control and Prevention (CDC).

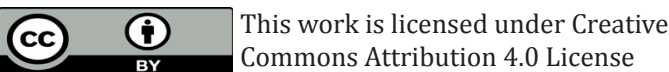

To Submit Your Article Click Here:

Submit Article

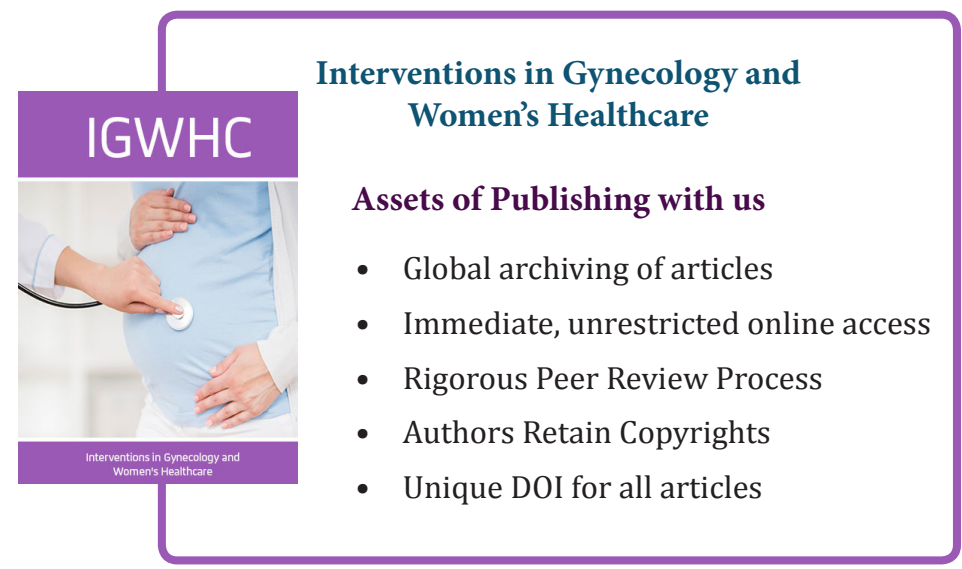

